\title{
La didáctica crítica: una opción pedagógica para la universidad de hoy.
}

\author{
Martha Cecilia Andrade Calderón ${ }^{1}$ \\ Clarena Muñoz Dagua ${ }^{1}$
}

\begin{abstract}
Resumen
Si bien la universidad colombiana en la práctica no ha desarrollado de manera amplia el concepto de didáctica para la educación superior, las facultades de educación sí han incursionado en las didácticas específicas de algunas disciplinas como las matemáticas, las ciencias naturales, el lenguaje, la literatura, la lectura y la escritura, entre otras. La discusión del tema se ha dado en espacios académicos del orden nacional e internacional en donde se ha dirimido sobre los conceptos de pedagogía y didáctica que, en su contextualización, permiten avizorar marcos y espectros generales que van desde los repasos de la escuela tradicional, pasando por la escuela nueva hasta la actual, que incluyen esta última pedagogías de apertura, como la activa y la crítica. Desde esta perspectiva, en la disertación nos enfocamos en la didáctica crítica y desde la mediación del lenguaje en la alternativa del taller crítico, como una opción pedagógica, oportuna, adecuada y pertinente para la universidad de hoy.
\end{abstract}

Palabras clave: didáctica crítica, investigación, pedagogía crítica, taller crítico. 


\title{
Critical Didactics: a pedagogic option for modern today's university.
}

\begin{abstract}
While Colombian university in practice broadly developed the concept of teaching for higher education, other education faculties have entered the specific teaching of some subjects such as mathematics, natural sciences, language, literature, reading and writing, among others. The discussion of the subject has been in academic areas of national and international order where it has settled on the concepts of pedagogy and didactics, in its contextualization, allow envision frames and general spectra ranging from traditional school rehearsals, passing for the new school to the present, including the latter opening pedagogies such as active and criticism. From this perspective, the dissertation we focus on the critical teaching and from the mediation of language in the alternative critical workshop as a teaching option, timely, appropriate and relevant for college today.
\end{abstract}

Key words: critical didactics, research, critical pedagogy, critical workshop.

Recibido: 16 de octubre 2012

Aceptado: 20 de diciembre 2012

\section{Punto de partida}

La discusión de la didáctica para la educación superior es un tema que ha sido abordado en espacios académicos del orden nacional e internacional. De hecho, al examinar las aplicaciones que se han dado de las propuestas, encontramos que en la práctica, en lo que corresponde a la universidad colombiana, son las facultades de educación las que han incursionado en las didácticas específicas de algunas disciplinas; pero a nivel general no hay unos criterios y argumentos claros para pensar una didáctica que permita contextualizar una enseñanza- aprendizaje pertinente a unos condicionamientos sociales, políticos y educativos particulares. Desde esta perspectiva, en esta disertación nos enfocamos en ofrecer algunos argumentos para plantear la didáctica crítica como una opción pedagógica 
pertinente para la universidad de hoy. Nos interesa compartir la experiencia como docentes de Lengua, a partir del Taller Crítico, el cual consideramos una estrategia oportuna, adecuada y pertinente para la formulación e implementación de una política educativa tendiente a centralizar la lectura y escritura como eje transversal de formación en las instituciones de educación superior.

Esta propuesta implica la introducción de tres aspectos fundamentales que aunque, en la práctica, se dan simultáneamente, por razones metodológicas desarrollamos de forma separada: la pedagogía crítica, la didáctica activacrítica y el Taller Crítico.

\section{En el camino de la didáctica crítica}

\section{La Pedagogía Crítica}

La ruta de la didáctica crítica tiene sus primeros indicios en la Pedagogía Crítica, la cual emerge como resultado de los trabajos de la Teoría Crítica en las décadas de los ochenta y los noventa. Algunos de sus fundamentos nacen de los filósofos y teóricos sociales de la escuela de Frankfurt, Alemania, quienes en sus reflexiones dieron la apertura a una nueva manera de leer la realidad, capaz de responder a las problemáticas sociales del mundo moderno. Según Peter McLaren (1997:125), "la pedagogía crítica examina a las escuelas tanto en su medio histórico como en su medio social por ser parte de la hechura social y política que caracteriza a la sociedad dominante". Se interesa en primer lugar, en una crítica a las estructuras sociales que afectan la vida de la escuela, particularmente situaciones relacionadas con la cotidianidad escolar y la estructura del poder. En segundo lugar, llama la atención al desarrollo de las habilidades de pensamiento crítico-reflexivo con el fin de transformar la sociedad. Es decir, parte del reconocimiento de la realidad, sus características, problemas, expectativas e intereses.

La Pedagogía Crítica, o también llamada Pedagogía social, aparece entonces como una propuesta alternativa, praxis liberadora, que pretende provocar transformaciones en el sistema educativo con matices políticos. En Latinoamérica se considera como su fundador al brasilero Paulo Freire. Su pensamiento educativo fue considerado como un aporte fundamental para esta corriente; según Vidal A. (2011) ella no constituye un campo teórico unificado -estamos de acuerdo con ello- sino un cuerpo de ideas comunes formuladas y difundidas por autores tales como el citado Freire, Henry Giroux, Peter McLaren, Michael Apple, entre otros.

Lo cierto es que Paulo Freire (2006:24), da mucha importancia a la práctica educativa, en cuanto a "Pedagogía de la autonomía. Saberes necesarios para la práctica educativa", lo que significa que la práctica educativa debe presentar los saberes que considera indispensables en su praxis, por eso hace un llamado a 
los educadores progresistas para que promuevan una pedagogía crítica fundada en la ética y el respeto por la dignidad y autonomía del educando. Asimismo, el brasilero Henry Giroux y otro (1990:177), propone que los docentes inscritos en la pedagogía crítica pueden adquirir la categoría de intelectuales transformativos para lograr que lo pedagógico sea más político y lo político más pedagógico, lo que representa incluir dentro del proceso pedagógico la política, en tanto que representa una lucha por la determinación de significados en un contexto de relaciones de poder; centra su trabajo en la propuesta de la labor intelectual de los maestros, las teorías de la reproducción y la pedagogía de la oposición.

Es relevante decir que el docente en la pedagogía crítica es el agente activo, dinámico que propicia relaciones entre los sujetos y su realidad en un esfuerzo por comprender esas relaciones $\mathrm{y}$, que descubre relaciones existentes entre poder, conocimiento y dominación. Esta pedagogía crítica mueve tanto al docente, líder del acto educativo, como a su coequipero el estudiante hacia un cuestionamiento propositivo de su realidad a buscar caminos emancipadores que coadyuven al progreso de una nación; consideramos, en definitiva, que esta es un buen camino, sin ser militancia política, para transformar y superar brechas sociales que lleven a la re-construcción de un país, porque la educación sin compromisos políticos -no politiqueros-, por esencia propia debe ser un acto liberador, revolucionario, per se, que permite cambiar al educando y por extensión su contexto.

De igual manera, se observa como la pedagogía crítica no riñe con las propuestas de la pedagogía activa que se caracterizan porque el aprendizaje debe darse desde las necesidades del estudiante y su contexto, lo cual compromete una educación funcional, acertada para cualquier nación. Ella, desde nuestra apreciación, es un buen nicho para la Educación Superior que debe fomentar procesos de aprendizaje comprometidos desde la crítica a la proposición de soluciones de problemas contextualizados. La Pedagogía Crítica facilita el evaluar tanto lo aprendido, desde la indagación, la duda y la sospecha; como el hallar nuevas apreciaciones y soluciones a problemas cognitivos y sociales que luego se visibilizarán en el ejercicio profesional con un alto valor argumentativo y académico.

Estos aspectos pedagógicos serán evidentes en el quehacer del aula universitaria siempre y cuando se le otorguen didácticas correspondientes, que ofrezcan respuestas al cómo hacer para enseñar y aprender con más eficacia en la universidad.

\section{El hacer de la didáctica: la didáctica activa-didáctica crítica}

La noción de pedagogía como ciencia general de la formación del ser humano y desde sus múltiples caminos recorridos, llámese activa, conceptual, crítica, o hedonista, invita a las preguntas, ¿Cómo enseñar en la universidad? y ¿Cuál sería 
la didáctica general que requerimos en una época en la que nuestros estudiantes son nativos digitales, con lógicas fragmentadas y lenguajes multimodales que rechazan no sólo las pedagogías tradicionales sino también las didácticas ligadas al quehacer conservador y formalista con que quizás, muchos de los actuales maestros universitarios fuimos formados?

Al hacer un rápido recorrido histórico de la didáctica, desde el siglo XVII, con el alemán Wolfgan Ratke, pasando por Comenius, se observa cómo esta disciplina inscrita en el ámbito de la organización, de las reglas y del método para enseñar de manera eficaz ha ido transformándose y aún mejor flexibilizándose; se pasó de aquella vieja escuela tradicional caracterizada por su rigidez, represión y métodos complejos de enseñanza, a una más "nueva" o liberal que convirtió lo tedioso en ameno y el aprendizaje estático en uno progresivo, gradual y práctico, incluyendo no solo en él lo intelectual, sino también lo espiritual.

Luego hacia el siglo XIX, John Dewey, propuso que el aprendizaje debería darse a través de actividades democráticas, más que por medio de contenidos homogenizadores y métodos autoritarios. Después de la posguerra de siglo XX, en Estados Unidos, se ofreció un enfoque tecnicista para la didáctica, (Davini, 1996), en donde se preguntaba qué es lo que la escuela debe enseñar desde la racionalidad técnica del currículo. Esto permitió la implementación de métodos y procedimientos precisos y concretos; el modelo de planificación por excelencia de tal movimiento fue el llamado modelo de objetivos de conducta.

Frente a este tecnicismo se contrapone la Escuela Crítica en Ciencias Sociales, 1980, con autores como Bordieu, Adorno y Foucault, que dieron origen a la pedagogía crítica. Desde la epistemología crítica de las ciencias sociales, incluida la pedagogía, se comienza a pensar en fenómenos sociales dinámicos, grupales con significados forjados desde una subjetividad, para organizar desde la experiencia y desde los componentes ideológico-políticos que atravesaban las prácticas educativas, nuevos métodos didácticos, más abiertos, activos y flexibles para el aprendizaje.

Ahora bien, la actual necesidad de dar solución a los desafíos de la calidad y equidad de la educación superior, debe reflejarse en una didáctica que desarrolle en forma dinámica escenarios para la acción. Por ello es necesaria, junto a la didáctica activa, una didáctica crítica que permita motivar, valorar el contexto, el sentido de aprender y el carácter personal del aprendizaje; activar las competencias complejas para favorecer el cambio conceptual y evitar la memorización; fomentar el interactuar con pares en ambientes que favorezcan el trabajo cooperativo. Una didáctica que se ajuste perfectamente a los principios y objetivos misionales de la universidad, cuanto se dice que es ella la que debe generar personas autónomas y críticas que aporten, no solo a la solución de problemas de un país sino que sean capaces de mirar la ciencia, dar alternativas y proponer nuevas verdades que encausen una mejor calidad de vida en el planeta. 
Para Davini (1996), por ejemplo, el método de la didáctica crítica es hermenéutico porque utiliza la interpretación como forma de aprehensión de la totalidad en lo particular y permite, agregaríamos nosotras, desarrollar competencias comunicativas, propositivas, argumentativas e interpretativas tan esenciales para el estudiante. En este sentido, la actividad docente universitaria desde esta didáctica crítica, circunscrita también como una didáctica activa, precisa de tres aspectos importantes: un dominio de la disciplina, una actitud dialógica frente al mundo y un uso pertinente y evaluativo del saber. Ello asegura inducir al estudiante a concebir miradas diferentes, enjuiciadoras, autónomas, frente al conocimiento, y a tomar posturas propositivas en el contexto académico, social y cultural.

En este marco didáctico y como docentes de Lengua materna proponemos el Taller Crítico como estrategia oportuna, adecuada y pertinente para la formulación e implementación de una política educativa, tendiente a centralizar la lectura y escritura como eje transversal de formación en las instituciones de educación superior que aspiran a promover estudiantes con sentido crítico y de responsabilidad social.

\section{El Taller Crítico: opción para una política didáctica universitaria}

En el contexto de las políticas educativas, consideramos que toda propuesta pedagógica debe tener mínimo tres cualidades que son relevantes para la puesta en escena y el Taller da cuenta de estas, pues como estrategia es oportuno, adecuado y pertinente. Oportuno porque da respuesta a una problemática de aprendizaje que se ha evidenciado en los resultados de las evaluaciones de calidad en la educación superior, tales como los de las pruebas SABER -PRO en las cuales las estadísticas han demostrado la necesidad de fortalecer las competencias de lectura y escritura y, el taller sería el espacio ideal, laboratorio propicio para producir el discurso académico, a partir del hacer en contexto. Adecuado, porque la estrategia, inspirada en la praxis, es apropiada a las condiciones y circunstancias de los actuales formatos para leer y escribir de los nativos digitales quienes se han formado en lógicas multimodales que exigen nuevas metodologías, donde el estudiante sea un actor dinámico comprometido con su propio proceso de aprendizaje. Y pertinente, porque el concepto de Taller Crítico, corresponde a la naturaleza dual de la educación superior: el desarrollo del pensamiento crítico y la formulación de alternativas viables a problemas concretos.

En efecto, en su proceso comunicativo de impacto socio cultural, el Taller Crítico, desde nuestra perspectiva (Andrade y Muñoz, 2004), genera seis condiciones favorables para ser incluida como estrategia de trabajo en el aula universitaria, más cuando se requiere con urgencia formar educadores con pensamiento crítico que coadyuven a los estudiantes a leer e interpretar con sentido crítico las disciplinas desde un saber hacer que reivindique la esencia del Taller en el aula universitaria: 
1. Comunicación multidimensional en el proceso, ya que debe tender a dar respuesta a las exigencias de conocimiento del grupo específico y la intención del acto discursivo, lo cualimplica la polifonía, escuchar, hablar y, a la vez, promover la inclusión de múltiples voces que den cuenta de lectura y la escritura.

2. Generación de conocimientos significativos y socialmente útiles, de tal manera que se genere el saber hacer en contexto.

3. Promoción de la investigación formativa, con la puesta en escena de formas didácticas que posibilitan el aprendizaje sobre la base del planteamiento de problemas que permiten el desarrollo de competencias argumentativas y propositivas por cuanto el estudiante prepara sus intervenciones con el fin de aportar al desarrollo de un verdadero laboratorio de investigación.

4. Autonomía para la construcción de conocimientos propios, pues el participante del acto comunicativo abstrae significados con una visión diferente para convertirse en un profesional capaz de lograr su desarrollo personal, seleccionando el saber que considera útil y valorando la información y el conocimiento de acuerdo con las dinámicas de su quehacer individual y el clima organizacional; esto genera en el aula de clase una puesta en escena del diálogo, la discusión y la confrontación de los coparticipantes.

5. Capacitación para aplicar la evaluación integral en las labores realizadas en la perspectiva de la autoevaluación, heteroevaluación y coevaluación como maneras de asumir críticamente el aprendizaje.

6. Efectividad en el proceso educativo en tanto la cualificación del estudiante se puede evidenciar en su participación, con la orientación del docente, en estrategias de interacción en el aula que le exigen poner a prueba sus lecturas textuales y contextuales, conocimientos, habilidades, destrezas, actitudes, valores, y expectativas, los cuales son socializados y confrontados en el contexto objetivo de circulación y producción de conocimientos.

Los resultados que otorga el ejercicio de una didáctica crítica en el aula universitaria, referidos a aspectos fundamentales como son la autonomía, investigación, resolución de problemas y la evaluación integral, nos indican que son asidero ideal para cumplir con los objetivos de la educación superior.

La didáctica crítica y desde nuestra perspectiva, el Taller Crítico, plantea para la universidad $\mathrm{o}$, de cara a las políticas públicas, una alternativa educativa activa, formadora, incluso, interdisciplinaria, por la cual el estudiante aprende a aprender, aprende a ser, aprende hacer y adquiere habilidades para leer, interpretar, sistematizar, elaborar y aplicar el conocimiento no sólo en el aspecto estrictamente académico sino en todo momento importante de su vida, aspecto 
fundamental para el aprendizaje que realmente se requiere. Por su parte, el profesor tallerista, llega con una propuesta oportuna, adecuada y pertinente para enseñar-aprendiendo, o aprender enseñando, y se transforma en un promotor de un proceso de formación para la vida, para el hombre que la sociedad necesita; un impulsador del intercambio de experiencias y puntos de vista en un escenario donde el diálogo potencia las posibilidades de aprender y construir el conocimiento y las soluciones en equipo.

En suma, consideramos que en la formulación de políticas educativas, se debe tener en cuenta estrategias metodológicas como el Taller Crítico que, en el marco de la pedagogía y la didáctica crítica, contribuyan a que el estudiante comprenda la realidad, influya en ella y tenga la posibilidad de actuar con el fin de transformar para mejorar. El éxito estará en que los estudiantes puedan y quieran realizar las operaciones cognitivas convenientes e interactúen adecuadamente con sus guías y los recursos educativos puestos a su alcance y, desde su autonomía, ofrezcan salidas críticas oportunas, adecuadas y pertinentes.

\section{Referencias bibliográficas}

Andrade C. Martha C y Muñoz D. Clarena. (2004). El taller crítico: una propuesta de trabajo interactivo. Revista tabula rasa. En revista de humanidades no. 2, enerodiciembre. Universidad Colegio Mayor de Cundinamarca. Bogotá.

Beard R. (1974). Pedagogía y didáctica de la Enseñanza Universitaria Oikos-Tau S. A. Barcelona.

Borja, Mónica. (2005). La pedagogía crítica y la contextualización de la enseñanza. hhtmIMónicaBorja.http://www.uninorte.edu.co/divisiones/iese/lumen/ediciones/4/ articulos/monica_borja.htm

Bourdieu, Pierre. (2008). Capital cultural, escuela y espacio social. siglo XXI editores. Buenos aires.

Davini et al. (1996). Corrientes didácticas contemporáneas. Buenos Aires: Editorial Paidós.

Demera Arango, Jaime. (2007). Hacia una didáctica crítica (II). Disponible en: http:// www.eldiario.com.ec/noticias-manabi-ecuador/100602-hacia-una-didactica-critica-ii/.

Facione, Peter A. \&Facione, N .C. (1992). The California Critical Thinking Dispositions Inventory (CCTDI) and the CCDTI test manual. Millbrae, California Academic Press. California.____.2003. Critical Thinking: What It Is and Why It Counts. (en inglés, formato PDF) http://www.calpress.com/pdf_files/what\&why.pdf. 
Freire, Paulo. (2006). Pedagogía de la Autonomía. Saberes necesarios para la práctica educativa. México, Siglo XXI Argentina.

Giroux, Henry. (1992). Teoría y resistencia en educación. Siglo XXI, UNAM. México. .Y MCLAREN, Peter. 1990 La Educación del profesor y la política de reforma democrática, y Los profesores como intelectuales. Hacia una pedagogía crítica del Aprendizaje. Centro de Publicaciones del Ministerio de Educación y Ciencia y Ediciones Paidós Ibérica S.A., pp. 209-227. Madrid.

McLaren, Peter. (1997). Pedagogía crítica y cultura depredadora. Políticas de oposición en la era posmoderna, Ed. Paidós (trad. al español por Pilar Pineda Herrero). Barcelona,

Muñoz D. Clarena, Andrade C., Martha, Cisneros, Mireya.(2011). Estrategias de interacción oral en el aula. Una didáctica crítica del Discurso educativo. Magisterio. Bogotá.

Paulo Freire . (2005). Pedagogía del Oprimido, Siglo XXI. México.

Vidal A. Leonardo. (2011). Intelectuales latinoamericanos: desfases, tensiones y proyecciones. Disponible en: http://www.usergioarboleda.edu.co/civilizar/civilizar-20/ INTELECTUALES\%2OLATINOAMERICANOS....pdf 